\title{
Coherent states in a laser cavity
}

\author{
K. M. Priyashanka, K. A. I. L Wijewardena Gamalath \\ Department of Physics, University of Colombo, Colombo3, Sri Lanka \\ E-mail address: imalie@phys.cmb.ac.lk
}

\begin{abstract}
Keywords: Coherent states, Fokker-Plank equation, density operator, resonant cavity
\end{abstract}
\begin{abstract}
A mathematical model was developed to obtain the population inversion of the atoms in laser field in a laser cavity by considering the electric field in the optical cavity and the atomic states of the medium to be quantized. The master equation of the density operator of the laser field was studied analytically and numerically. Using coherent states, the Fokker-Plank equation for the phase space density for the laser field was solved analytically for the time dependent and steady state situations. The laser field above the threshold can be represented by a randomly phased mixture of coherent states. As the pump parameter increases in the laser process, the phase density becomes narrower, tending toward a delta function, creating a coherence laser field.
\end{abstract}

\section{INTRODUCTION}

In 1917 Albert Einstein established the foundation for the laser suggesting the existence of the phenomena of stimulated emission [1]. This phenomenon of stimulated emission was used for the amplification of the electromagnetic field, and then to combine the amplifier with a resonator to make an oscillator [2]. The theoretical studies of lasers are mainly based on quantum field theory and quantum optics. The concept of coherent states, first introduced by Glauber [3] plays a major role in laser physics. Quantum coherent states are not strongly related to the classical term coherence but refer to a special kind of pure quantum-mechanical state of the light field corresponding to a single resonator mode. In some respect, the coherent state has properties which are relatively close to a classical state of the light field, resembling a classical oscillation of the light field. Laser radiation is produced in a resonant cavity where the resonant frequency of the cavity is the same as the frequency associated with the atomic electron transitions providing energy flow into the field. As energy in the resonant mode builds up, the probability for stimulated emission in that mode only, increases. In a laser the light emitted in to this resonant state is highly coherent. Glauber [3] provided a complete quantum-theoretical description of coherence in the electromagnetic field. The density operator of a single mode laser field can be defined as an integration of averaged outer products of coherent states over entire complex plane. The coherent state representation of the laser field can be reduced to the Fokker-Plank equation of phase space density by introducing the coefficients for gain, loss and saturation of the laser field [4]. Electromagnetic field established in the optical cavity of a single-mode laser can be represented by the coherent state, using semi classical and quantum mechanical models [5].

In this paper, the properties and behaviour of an optical resonant cavity due to a laser operating in a single mode is studied using quantum theory and coherent state representation. The electric field in the optical cavity and the atomic states of the medium were considered to be quantized. A simple mathematical model for population inversion of the atoms in laser medium was obtained and the master equation of the density operator of the laser field was studied analytically and numerically. In the treatment using coherent states the Fokker-Plank equation for the phase space density for the laser field was solved analytically for both time dependent situation and steady state situation. A numerical solution is presented for the time dependent solution. 


\section{PHTONS INSIDE THE RESONANCE CAVITY AT STEADY STATE}

In a quantum mechanical treatment of a laser field in an optical cavity, the electric field is considered to be quantized. The quantized field treatment provides a more consistent foundation for the laser theory. The single mode electric field operator inside an empty cavity can be written as,

$$
\boldsymbol{E}(\boldsymbol{r}, t)=\hat{e} \sqrt{\frac{\hbar \omega}{2 V \varepsilon_{0}}}\left[\hat{a} U(\boldsymbol{r}) e^{-i \omega t}+\hat{a}^{\dagger} U^{*}(\boldsymbol{r}) e^{+i \omega t}\right]
$$

where $V$ is the volume of the cavity, the normalized cavity mode function $U(\boldsymbol{r})$ for an optical cavity formed by plane mirrors at $z=0$ and $z=L$ can be written as $U(\boldsymbol{r})=e^{i k z}(k=n \pi c / L)$ and $\hat{e}$ is a real unit polarization vector representing a linear polarized wave. The operators $a$ and $a^{\dagger}$ represent the annihilation and creation operators respectively. If $|n\rangle$ is the state corresponding to $n$ number of photons, then the annihilation and creation operators give,

$$
\hat{a}|n\rangle=\sqrt{n}|n-1\rangle, \quad \hat{a}^{\dagger}|n\rangle=\sqrt{n+1}|n+1\rangle
$$

If the atomic population is inverted in a set of identical two-level atoms interacting with the field of a single cavity mode, then the coupling between atoms and the field may cause the number of photons in the cavity mode to grow in time. The Hamiltonian of the coupled system consisting of a single-mode electromagnetic field of frequency $\omega$ and a two-level atoms of resonance frequency $\omega_{0}$ located at position $\boldsymbol{r}$ may be taken to be formed from atomic Hamiltonian $H_{A}$, field Hamiltonian $H_{F}$ and the interaction Hamiltonian $H_{I}[4]$,

$$
H=H_{A}+H_{F}+H_{I}
$$

where

$$
\begin{aligned}
& H_{A}=E_{0}+\frac{\hbar \omega_{0}}{2}\left(\hat{b}^{\dagger} \hat{b}-\hat{b} \hat{b}^{\dagger}\right) ; \quad H_{F}=\hbar \omega\left(\hat{n}+\frac{1}{2}\right) \\
& H_{I}=-\hat{\mu}_{12} \boldsymbol{E}(\boldsymbol{r}, t)\left(\hat{b}(t)+\hat{b}^{\dagger}(t)\right)
\end{aligned}
$$

$\hat{n}$ represents the photon number operator, $E_{0}$ the average energy of the two laser levels, $\hat{\mu}_{12}$ is the transition dipole moment of the atom and $\hat{b}$ and $\hat{b}^{\dagger}$ represent the atomic lowering and raising operators respectively. The atomic lowering and raising operators evolve in time according to the relations:

$$
\hat{b}(t)=\hat{b} e^{-i \omega_{0} t} ; \quad \hat{b}^{\dagger}(t)=\hat{b}^{\dagger} e^{i \omega_{0} t}
$$

If the interaction is turned on at the time $t_{0}$, with the single mode electric field operator given in equation 1 , then the interaction term given in equation 4 read,

$$
H_{I}=-\hbar g\left(\hat{a} \hat{b} U(\boldsymbol{r}) e^{-i\left(\omega+\omega_{0}\right) t}+\hat{a} \hat{b}^{\dagger} U(\boldsymbol{r}) e^{-i\left(\omega-\omega_{0}\right) t}+\hat{a}^{\dagger} \hat{b}^{\dagger} U^{*}(\boldsymbol{r}) e^{i\left(\omega+\omega_{0}\right) t}+\hat{a}^{\dagger} \hat{b} U^{*}(\boldsymbol{r}) e^{i\left(\omega-\omega_{0}\right) t}\right)
$$


where $g=\left(\omega / 2 \hbar V \varepsilon_{0}\right)^{1 / 2} \hat{\mu}_{12} \cdot \hat{e}$ is the coupling constant characterizing the strength of the interaction [5]. We can express the density operator $\hat{\rho}$ in terms of reduced density operators of the atom $\hat{\rho}_{A}$ and the field $\hat{\rho}_{F}$

$$
\hat{\rho}\left(t_{0}\right)=\hat{\rho}_{A}\left(t_{0}\right) \otimes \hat{\rho}_{F}\left(t_{0}\right)
$$

As a result of the interaction, density operator evolves in time in the interaction picture. The form of it at a later time $t$ can be obtained by a perturbation expansion. By regarding $\hat{\rho}\left(t_{0}\right)$ as the zeroth order approximation of $\hat{\rho}(t)$,

$$
\hat{\rho}^{(0)}(t)=\hat{\rho}\left(t_{0}\right)
$$

From the perturbation theory, the first order approximation reads:

$$
\hat{\rho}^{(1)}(t)=\hat{\rho}\left(t_{0}\right)+\frac{1}{i \hbar} \int_{t_{0}}^{t}\left[H_{I}\left(t_{1}\right), \hat{\rho}\left(t_{0}\right)\right] d t_{1}
$$

By repetition of the same procedure and taking the trace over the atomic variables, the effect of the interaction on the state of the laser field at time $t$ can be expressed in the form:

$$
\hat{\rho}_{F}(t)=\hat{\rho}_{F}\left(t_{0}\right)+t r_{A} \sum_{r=1}^{\infty} \frac{1}{(i \hbar)^{2}} \int_{t_{0}}^{t} d t_{1} \int_{t_{0}}^{t_{1}} d t_{2} \ldots \int_{t_{0}}^{t_{r-1}} d t_{r}\left[H_{I}\left(t_{1}\right),\left[H_{I}\left(t_{2}\right),\left[\ldots,\left[H_{I}\left(t_{r}\right), \hat{\rho}_{F}\left(t_{0}\right)\right]\right] \ldots\right]\right]
$$

In order to determine the effect of a single excited atom in the laser field, the initial atomic state is chosen as the upper state $\hat{\rho}_{A}\left(t_{0}\right)=|2\rangle\langle 2|$ and the interaction is allowed to proceed for a time up to an order of the lifetime of the upper state. The two laser levels $|1\rangle$ and $|2\rangle$ are assumed to decay to other states with average lifetime $T_{1}$ and $T_{2}$ respectively. The effect of an ensemble of such excited atoms on the field is therefore obtainable at least approximately by choosing $t-t_{0}=\Delta t$ to be the atomic lifetime and averaging over the ensemble of $\Delta t$ with an exponential probability distribution of average $T_{2}$. The reduced field density operator obtained in equation 10 is used to obtain the density operator given in equation 7 . It is clear that if the lifetime $\Delta t$ is very long compared with the optical period $2 \pi / \omega_{0}$, then the terms $\exp \left( \pm i\left(\omega+\omega_{0}\right) t\right)$ in equation 10 will integrate almost to zero and can be neglected. On the other hand, the contributions made by the terms in $\exp \left( \pm i\left(\omega-\omega_{0}\right) t\right)$ depend strongly on the magnitude of the term $\left(\omega-\omega_{0}\right)$. For simplicity we have assumed that this term is so small that, $\left|\left(\omega-\omega_{0}\right)\right| \Delta t \square 1$ which allows us to neglect the oscillatory factors altogether and treat $H_{I}$ as time-independent under the integral. Finally averaging over the lifetime $\Delta t$ with the exponential probability distribution, $P(\Delta t)=\left(e^{-\Delta t / T_{2}}\right) / T_{2}$, the change in the reduced field density operator can be written as,

$$
\begin{aligned}
\Delta \hat{\rho}_{F}\left(t_{0}\right)= & -\left(g T_{2}\right)^{2}|U(r)|^{2}\left[\hat{a} \hat{a}^{\dagger} \hat{\rho}_{F}\left(t_{0}\right)-\hat{a}^{\dagger} \hat{\rho}_{F}\left(t_{0}\right) \hat{a}+\text { h.c. }\right] \\
& +\left(g T_{2}\right)^{4}|U(r)|^{4}\left[\hat{a} \hat{a}^{\dagger} \hat{a} \hat{a}^{\dagger} \hat{\rho}_{F}\left(t_{0}\right)+3 \hat{a} \hat{a}^{\dagger} \hat{\rho}_{F}\left(t_{0}\right) \hat{a} \hat{a}^{\dagger}-4 \hat{a}^{\dagger} \hat{a} \hat{a}^{\dagger} \hat{\rho}_{F}\left(t_{0}\right) \hat{a}+\text { h.c. }\right]
\end{aligned}
$$

h.c. represent the Hermitian conjugate. A real laser has many excited atoms and molecules distributed throughout the active medium which are excited by some pumping mechanism at a 
certain rate $R_{2}$. The contribution to the change of $\hat{\rho}_{F}$ made by any one of them is therefore small, and if the laser field evolves slowly, the change $\hat{\rho}_{F}\left(t_{0}\right)$ given in equation 11 can be considered as a good approximation. The average rate of change of the density operator of the laser field brought about by gain mechanism is,

$$
\begin{aligned}
\left(\frac{\partial \hat{\rho}_{F}}{\partial t}\right)_{\text {gain }}= & -\frac{1}{N} R_{2}\left(g T_{2}\right)^{2} \int \eta(\boldsymbol{r})|U(\boldsymbol{r})|^{2}\left[\hat{a} \hat{a}^{\dagger} \hat{\rho}_{F}+\hat{a}^{\dagger} \hat{\rho}_{F} \hat{a}+h . c .\right] d^{3} r \\
& +\frac{1}{N} R_{2}\left(g T_{2}\right)^{4} \int \eta(\boldsymbol{r})|U(\boldsymbol{r})|^{4}\left[\hat{a} \hat{a}^{\dagger} \hat{a} \hat{a}^{\dagger} \hat{\rho}_{F}+3 \hat{a} \hat{a}^{\dagger} \hat{\rho}_{F} \hat{a} \hat{a}^{\dagger}-4 \hat{a}^{\dagger} \hat{a} \hat{a}^{\dagger} \hat{\rho}_{F} \hat{a}+\text { h.c. }\right] d^{3} r
\end{aligned}
$$

Here $\eta(\boldsymbol{r})$ is the density of active laser atoms, $N$ is their total number and the integral is taken over entire cavity volume. The effect of loss on field density operator induced by absorption of an atom in lower state with $N_{1}$ atoms with $R_{1}$ density present in the laser cavity is

$$
\left(\frac{\partial \hat{\rho}_{F}}{\partial t}\right)_{\text {loss }}=-\frac{1}{N_{1}} R_{1}\left(g T_{1}\right)^{2} \int \eta_{1}(\boldsymbol{r})|U(\boldsymbol{r})|^{2}\left[\hat{a} \hat{a}^{\dagger} \hat{\rho}_{F}+\hat{a}^{\dagger} \hat{\rho}_{F} \hat{a}+\text { h.c. }\right] d^{3} r
$$

The master equation for the density operator $\hat{\rho}_{F}$ of the laser field can be obtained by combining the effects of the gain $A$ and the loss $C$ contributions

$$
\begin{aligned}
\frac{\partial \hat{\rho}_{F}}{\partial t}= & -\frac{1}{2} A\left[\hat{a} \hat{a}^{\dagger} \hat{\rho}_{F}+\hat{a}^{\dagger} \hat{\rho}_{F} \hat{a}+h . c .\right]-\frac{1}{2} C\left[\hat{a} \hat{a}^{\dagger} \hat{a} \hat{\rho}_{F}+\hat{a} \hat{\rho}_{F} \hat{a}^{\dagger}+\text { h.c. }\right] \\
& -\frac{1}{8} B\left[\hat{a} \hat{a}^{\dagger} \hat{a} \hat{a}^{\dagger} \hat{\rho}_{F}+3 \hat{a} \hat{a}^{\dagger} \hat{\rho}_{F} \hat{a} \hat{a}^{\dagger}-4 \hat{a}^{\dagger} \hat{a} \hat{a}^{\dagger} \hat{\rho}_{F} \hat{a}+\text { h.c. }\right]
\end{aligned}
$$

The gain $A$, the loss $C$ and the nonlinearity or the saturation $B$ of the laser are given by

$$
\begin{aligned}
& A=\frac{2 R_{2}}{N}\left(g T_{2}\right)^{2} \int \eta(\boldsymbol{r})|U(\boldsymbol{r})|^{2} d^{3} r \\
& B=\frac{8 R_{2}}{N}\left(g T_{2}\right)^{4} \int \eta(\boldsymbol{r})|U(\boldsymbol{r})|^{4} d^{3} r \\
& C=\frac{2 R_{1}}{N_{1}}\left(g T_{1}\right)^{2} \int \eta_{1}(\boldsymbol{r})|U(\boldsymbol{r})|^{2} d^{3} r
\end{aligned}
$$

where $U(\boldsymbol{r})$ is the normalized cavity mode function and $g=\sqrt{\omega / 2 \hbar \varepsilon_{0} V} \hat{\mu}_{12} . \hat{e}$. If $P(n, t)$ is the probability that there are $n$ photons in the laser cavity at time $t$, then $P(n, t)=\left\langle n\left|\hat{\rho}_{F}\right| n\right\rangle$ can be obtained by taking inner product of equation 14

$$
\begin{array}{r}
\left\langle n\left|\frac{\partial \hat{\rho}_{F}}{\partial t}\right| n\right\rangle=-\frac{1}{2} A\left\langle n\left|\left(\hat{a} \hat{a}^{\dagger} \hat{\rho}_{F}+\hat{a}^{\dagger} \hat{\rho}_{F} \hat{a}+h . c .\right)\right| n\right\rangle-\frac{1}{2} C\langle n|\left(\hat{a}^{\dagger} \hat{a} \hat{\rho}_{F}+\hat{a} \hat{\rho}_{F} \hat{a}^{\dagger}+\text { h.c. }\right)|n\rangle \\
-\frac{1}{8} B\langle n|\left(\hat{a} \hat{a}^{\dagger} \hat{a} \hat{a}^{\dagger} \hat{\rho}_{F}+3 \hat{a} \hat{a}^{\dagger} \hat{\rho}_{F} \hat{a} \hat{a}^{\dagger}-4 \hat{a}^{\dagger} \hat{a} \hat{a}^{\dagger} \hat{\rho}_{F} \hat{a}+\text { h.c. }\right)|n\rangle
\end{array}
$$

From equation 2: 


$$
\begin{aligned}
& \frac{\partial}{\partial t} P(n, t)=-A[(n+1) P(n, t)+n P(n-1, t)]-C[n P(n, t)+(n+1) P(n+1, t)] \\
&+B\left[(n+1)^{2} P(n, t)-n^{2} P(n-1, t)\right]
\end{aligned}
$$

For the steady state, $\frac{\partial}{\partial t} P(n, t)=0$ gives:

$$
A(n+1)\left(1-\frac{B}{A}(n+1)\right) P(n, t)-C(n+1) P(n+1, t)=A n\left(1-\frac{B}{A} n\right) P(n-1, t)-C n P(n, t)
$$

If $n$ is replaced by $(n-1)$ in the left hand side, then it is equivalent to the right hand side. Therefore both sides must be equal to zero and the recursion relation leads to:

$$
\begin{aligned}
& P(n)=\frac{A}{C}\left[1-\frac{B}{A} n\right] P(n-1)=p(0)\left(\frac{B}{C}\right)^{n} \prod_{j=1}^{n}\left(\frac{A}{B}-j\right) \\
& \text { For } B n / A \square 1: \quad P(n)=p(0)\left(\frac{A^{2}}{C B}\right)^{n} \frac{1}{\left(\frac{A}{B}+n\right) !}
\end{aligned}
$$

Considering the Stirling's approximation $n !=\sqrt{2 \pi} n^{n+1 / 2} e^{-n}$ for large $n$, so that $n^{2} \square n$ equation 20 can be written as,

$$
P(n)=\text { const. }\left(\frac{A}{C}\right)^{n} e^{-B n^{2} / 2 A}=\text { const. } e^{-(n \sqrt{2} / a-b)^{2} / 4}
$$

where $a^{2}=A / B$ and $A / C=e^{b / \sqrt{2} a}$. This is a Gaussian distribution. When $A>C(b>0)$, and $A<C(b<0)$. The mean value of $n$ can be found by

$$
<n>=\frac{\int_{0}^{\infty} n P(n) d n}{\int_{0}^{\infty} P(n) d n}=\frac{\int_{0}^{\infty} n e^{-(n \sqrt{2} / a-b)^{2} / 4} d n}{\int_{0}^{\infty} e^{-(n \sqrt{2} / a-b)^{2} / 4} d n}=\frac{a}{\sqrt{2}}\left\{\frac{2 e^{-b^{2} / 4}}{\sqrt{\pi}(1+\operatorname{erf}(b / 2))}+b\right\}
$$

The variance on $n$ is given by:

$$
\left\langle(\Delta n)^{2}\right\rangle=\left\langle n^{2}\right\rangle-\langle n\rangle^{2}=a^{2}\left[1-\frac{b e^{-b^{2} / 4}}{\sqrt{\pi}(1+\operatorname{erf}(b / 2))}-\frac{2 e^{-b^{2} / 2}}{\pi(1+\operatorname{erf}(b / 2))^{2}}\right]
$$

Figure 1 shows the probability of finding $n$ number of photons inside the laser cavity as a function of the photon number $n / a \sqrt{2}$ in the steady state for a pump parameter of $b=100$. The density distribution is a Poisson distribution around 100. Figure 2 shows the variation of the probability distribution of photons $P(n)$ in the optical cavity in the steady state for pump parameters $-1,0,1,2,7$. As the pump parameter $b$ moves from negative to positive value, the 
probability function moves right along the $x$ axis. The height of the probability density also changes, but however when the pump parameter exceeds 1 or 2 , the probability distribution is only a translation with negligible change of shape. For negative pump parameter, the probability distribution has an exponential variation and at the threshold $b=0$ , the slope of $P(n)$ at $n=0$ is zero. Figure 3 shows the probability distribution of photons $P(n)$ in the optical cavity in the steady state with pump parameter $b$ as well as photon number $n / a \sqrt{2}$ in three dimensions. For large pump parameters, the shape of photon

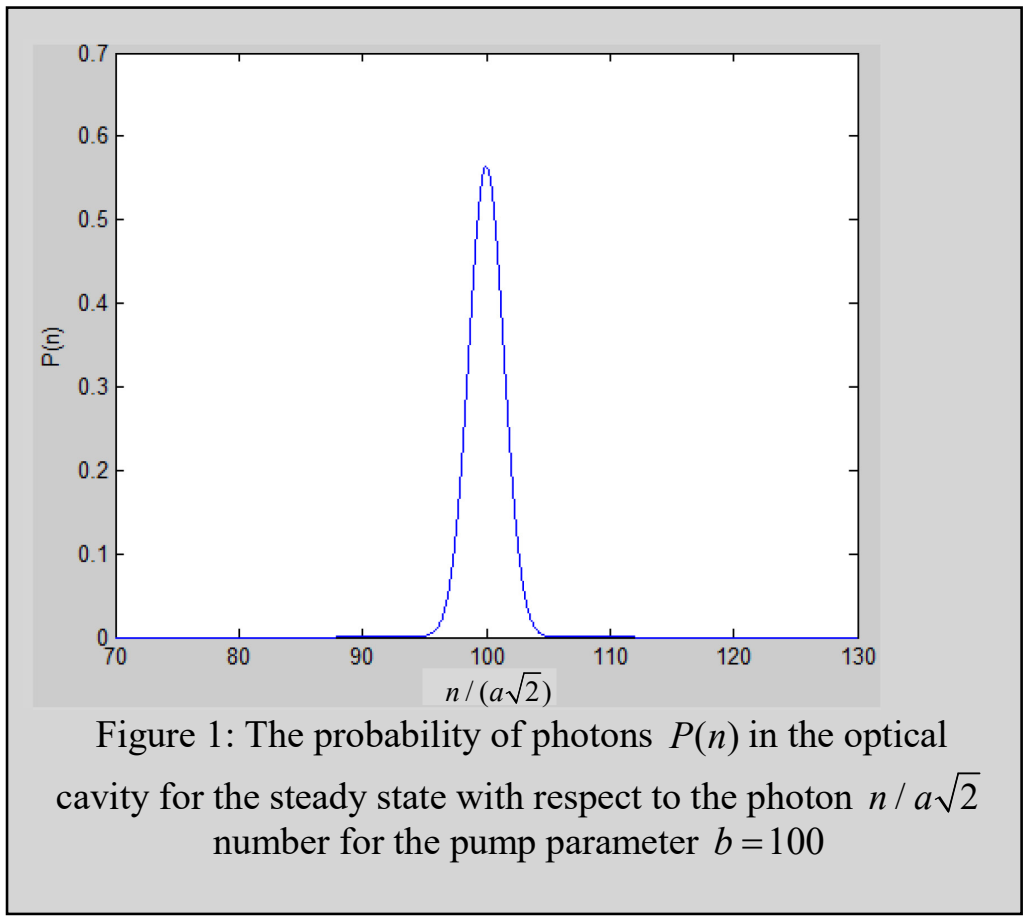
distribution in the optical cavity does not change much.

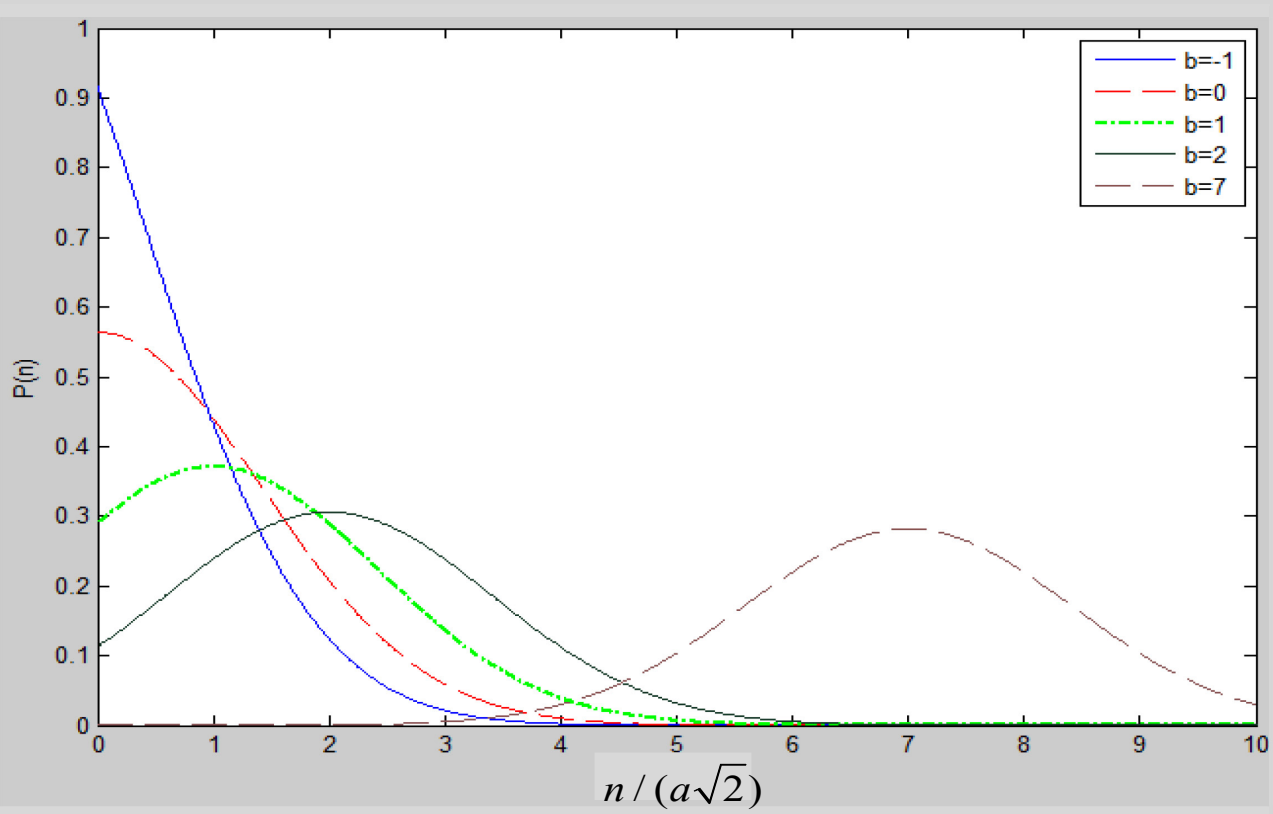

Figure 2: The probability distribution of photons $P(n)$ in the steady states for the pump parameter, $b=-1,0,1,2,7$ 


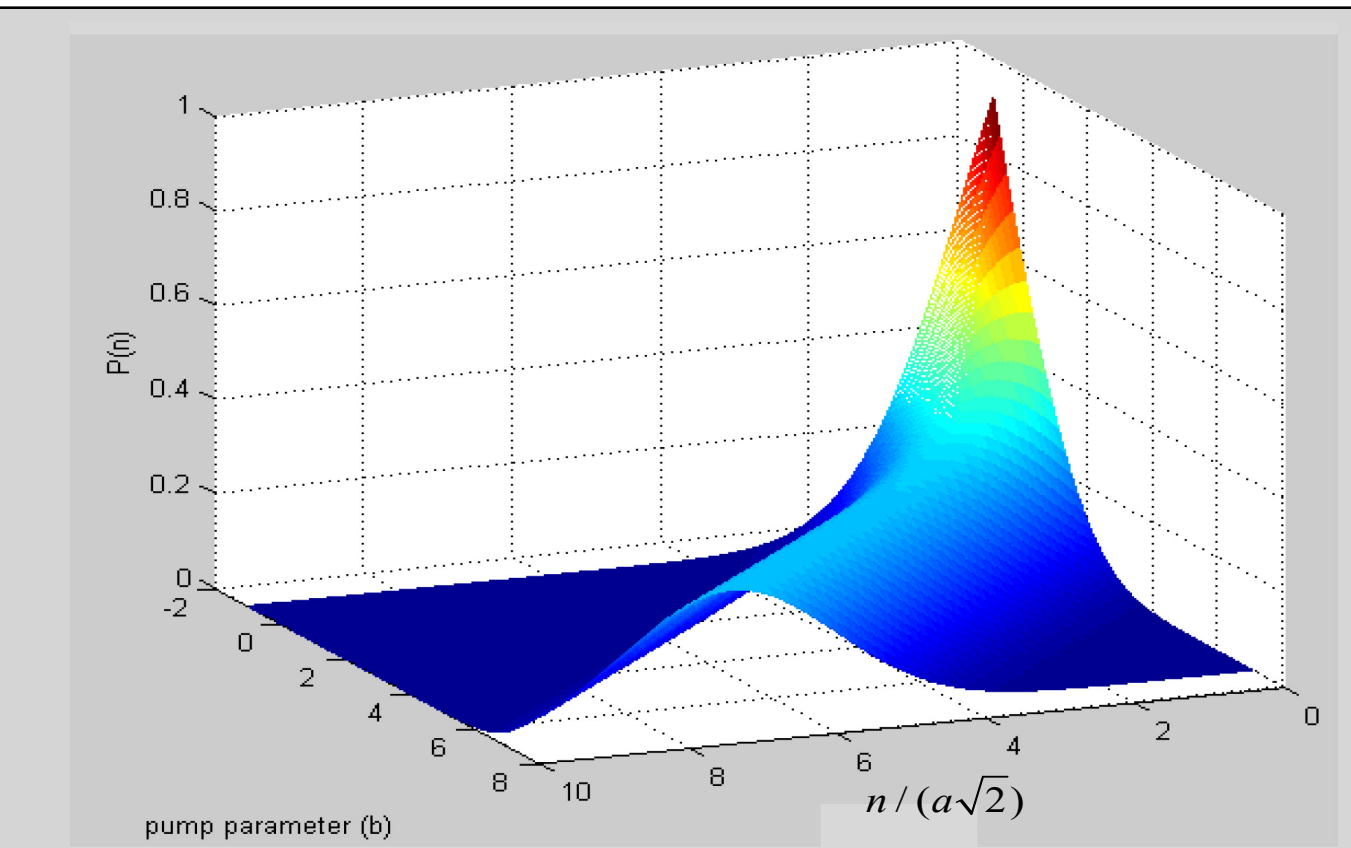

Figure 3: The probability distribution of photons $P(n)$ in the steady states for different values of the pump parameter $b$ and photon number $n$

The mean photon number $\langle n\rangle$ in the laser cavity in the steady state against the pump parameter $b$ is presented in figure 4. For negative pump parameter, $\langle n\rangle$ is not completely zero and there is no discontinuity at the origin that is at the threshold unlike in semi-classical theory, when the electric field in the optical cavity is treated classically and the energy states of atoms as quantized [6]. For positive pump parameter, the $\langle n\rangle$ value increases linearly with $b$. Figure 5 shows the variation of variance of the photon number $\left\langle\Delta n^{2}\right\rangle$ against the pump parameter $b$. For negative values of the pump parameter $\left\langle\Delta n^{2}\right\rangle$ is small but not zero. As the pump parameter increases positively, the $\left\langle\Delta n^{2}\right\rangle$ value increase

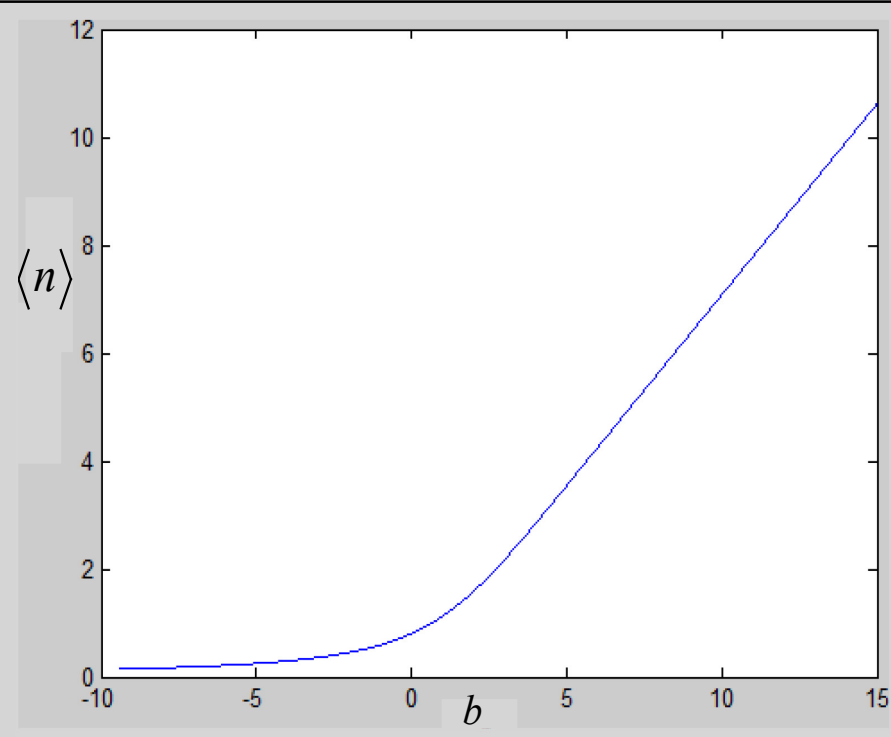

Figure 4: The mean photon number $\langle n\rangle$ in the laser cavity in the steady state with pump parameter $b$ dramatically and for larger values of $b$ it almost approaches its upper limit of 1 . The relative mean squared photon fluctuations $\left\langle(\Delta n)^{2}\right\rangle /\langle n\rangle^{2}$ with the pump parameter $b$ are presented in figure 6 . The variation of the relative mean square photon number goes to 1 as $b$ increases negatively and to zero as $b$ increase positively. For lager $b$ beyond 10, the relative mean squared photon fluctuations are almost zero. 


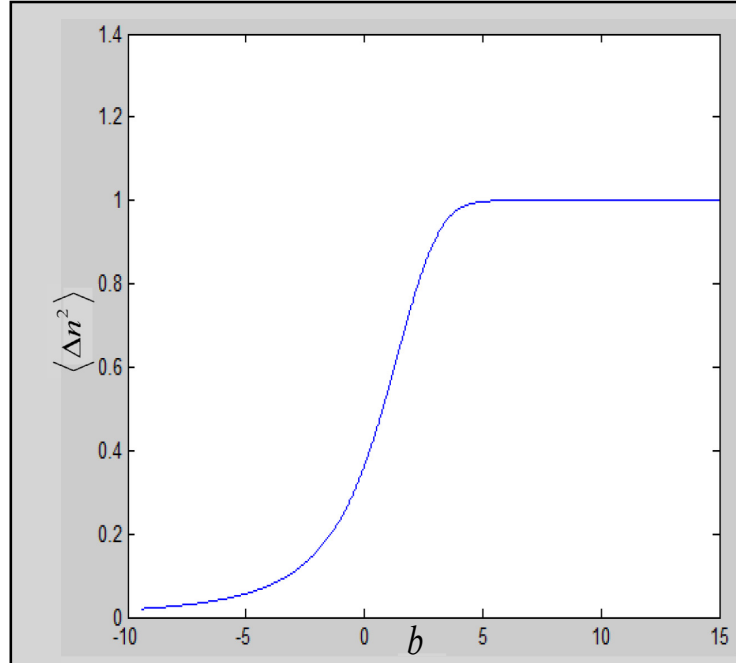

Figure 5: The variance of the photon photon fluctuations $\left\langle\Delta n^{2}\right\rangle$ against the pump parameter $b$ in the steady state.

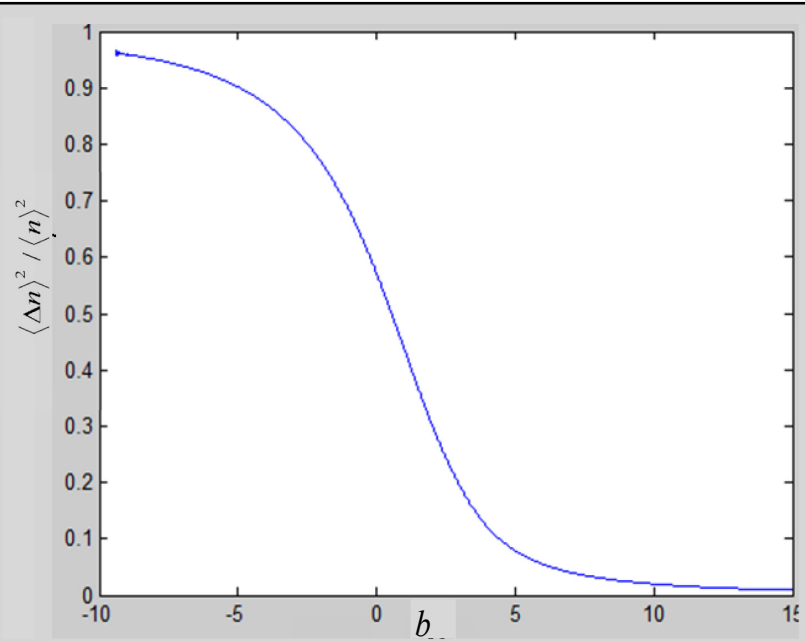

Figure 6: The relative mean squared photon fluctuations $\left\langle(\Delta n)^{2}\right\rangle /\langle n\rangle^{2}$ in the steady state with respect to pump parameter $b$

\section{PROBABILITY OF ABSORPTION AND EMISSION}

A laser with a medium of two level atoms or atomic dipoles located in the laser cavity interacting with a field treated as in a single cavity mode is considered. When the population inversion grows up, the coupling between field and the atoms may cause the number of photons in the cavity to grow up. The atom is initially in the lower energy state with $n+1$ number of photons. The atom-field system for absorption and emission is shown in figure 7. Atom absorbs a photon and transit in to the higher energy level. So the two quantum states for the atom and the field can be written as

$$
\begin{gathered}
\left|\psi_{1}\right\rangle=\left|\phi_{1}\right\rangle|n+1\rangle e^{-i E_{1} t / \hbar} \\
\left|\psi_{2}\right\rangle=\left|\phi_{2}\right\rangle|n\rangle e^{-i\left(E_{1} / \hbar+\omega_{0}\right) t} \\
\hat{b}\left|\phi_{1}\right\rangle=|0\rangle, \quad \hat{b}\left|\phi_{2}\right\rangle=\left|\phi_{1}\right\rangle \\
\hat{b}^{\dagger}\left|\phi_{1}\right\rangle=\left|\phi_{2}\right\rangle \quad \hat{b}^{\dagger}\left|\phi_{2}\right\rangle=|0\rangle .
\end{gathered}
$$

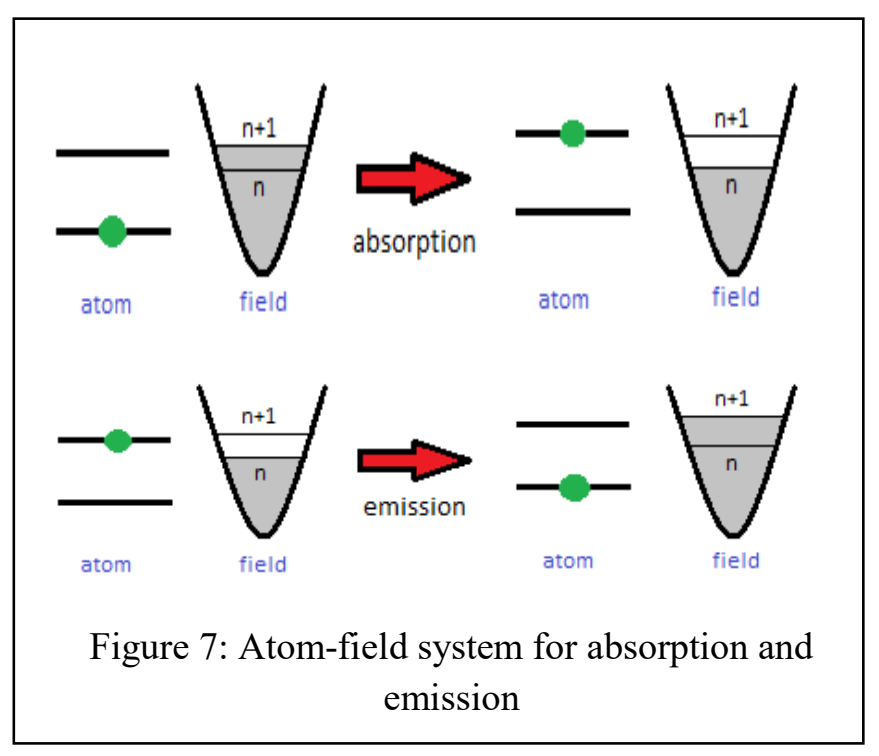

Let $|\psi\rangle$ be a linear combination of the two quantum states:

$$
|\psi\rangle=C_{1, n+1}(t)\left|\psi_{1}\right\rangle+C_{2, n}(t)\left|\psi_{2}\right\rangle
$$


From Schrodinger equation for the Hamiltonian of the coupled system

$$
\left[H_{A}+H_{F}+H_{I}\right]\left(C_{1, n+1}(t)\left|\psi_{1}\right\rangle+C_{2, n}(t)\left|\psi_{2}\right\rangle\right)=i \hbar \frac{\partial}{\partial t}\left(C_{1, n+1}(t)\left|\psi_{1}\right\rangle+C_{2, n}(t)\left|\psi_{2}\right\rangle\right)
$$

Substituting for $H_{A}$ and $H_{F}$ from equation 4:

$$
C_{1}(t) \hbar \omega\left(\hat{n}+\frac{1}{2}\right)\left|\psi_{1}\right\rangle+C_{2}(t) \hbar \omega\left(\hat{n}+\frac{1}{2}\right)\left|\psi_{2}\right\rangle+H_{I}|\psi\rangle=i \hbar\left(\dot{C}_{1}(t)\left|\psi_{1}\right\rangle+\dot{C}_{2}(t)\left|\psi_{2}\right\rangle\right)
$$

Since $\left|\psi_{1}\right\rangle$ and $\left|\psi_{2}\right\rangle$ are orthonormal, taking the inner product with $\left|\psi_{1}\right\rangle$ and $\left|\psi_{2}\right\rangle$ lead to:

$$
\begin{aligned}
& C_{1}(t) \hbar \omega\left(n+\frac{3}{2}\right)+\left\langle\psi_{1}\left|H_{I}\right| \psi\right\rangle=i \hbar \dot{C}_{1}(t) \\
& C_{2}(t) \hbar \omega\left(n+\frac{1}{2}\right)+\left\langle\psi_{2}\left|H_{I}\right| \psi\right\rangle=i \hbar \dot{C}_{2}(t)
\end{aligned}
$$

With the interaction Hamiltonian $H_{I}$ given in equation 6,

$$
\left\langle\psi_{1}\left|H_{I}\right| \psi\right\rangle=C_{1}(t)\left\langle\psi_{1}\left|H_{I}\right| \psi_{1}\right\rangle+C_{2}(t)\left\langle\psi_{1}\left|H_{I}\right| \psi_{2}\right\rangle
$$

the first term in the right hand side becomes zero since $\left\langle\phi_{n}|\hat{\alpha}| \phi_{n}\right\rangle=0 \quad\left(\hat{\alpha}=\hat{a}, \hat{a}^{\dagger}, \hat{b}, \hat{b}^{\dagger}\right)$ and the remaining term read:

$$
\left\langle\psi_{1}\left|H_{I}\right| \psi\right\rangle=C_{2}(t)\left\langle\psi_{1}\left|H_{I}\right| \psi_{2}\right\rangle
$$

$H_{I}$ is given in equation 6 and from equation 2 and 25 only the fourth term is nonzero. The above equation can be written as,

$$
\left\langle\psi_{1}\left|H_{I}\right| \psi\right\rangle=-C_{2}(t) \hbar g e^{-i \omega_{0} t}\left\langle n+1\left|\hat{a}^{\dagger}\right| n\right\rangle\left\langle\phi_{1}|\hat{b}| \phi_{2}\right\rangle U^{*}(\boldsymbol{r}) e^{i\left(\omega-\omega_{0}\right) t}=-C_{2}(t) \hbar g U^{*}(\boldsymbol{r}) e^{i\left(\omega-2 \omega_{0}\right) t} \sqrt{n+1}
$$

Similarly

$$
\left\langle\psi_{2}\left|H_{I}\right| \psi\right\rangle=C_{1}(t)\left\langle\psi_{2}\left|H_{I}\right| \psi_{1}\right\rangle=-C_{1}(t) \hbar g U(\boldsymbol{r}) e^{-i\left(\omega-2 \omega_{0}\right) t} \sqrt{n+1}
$$

Substituting the above results obtained in equations 32 and 33 in equation 29:

$$
\begin{aligned}
& i \dot{C}_{1}(t)=-A_{1} C_{2}(t) e^{i\left(\omega-2 \omega_{0}\right) t}+C_{1}(t) B_{1} \\
& i \dot{C}_{2}(t)=-A_{2} C_{1}(t) e^{-i\left(\omega-2 \omega_{0}\right) t}+C_{2}(t) B_{2}
\end{aligned}
$$

where $A_{1}=g U^{*}(\boldsymbol{r}) \sqrt{n+1}, A_{2}=g U(\boldsymbol{r}) \sqrt{n+1}, B_{1}=\omega(n+3 / 2), B_{2}=\omega(n+1 / 2) . \quad$ Differentiating second equation in equation 34 and substituting to the first leads to,

$$
\ddot{C}_{2}(t)+i a \dot{C}_{2}(t)-b C_{2}(t)=0
$$


where $a=\left(\omega-2 \omega_{0}+B_{1}+B_{2}\right)$ and $b=B_{2}\left(\omega-2 \omega_{0}\right)+B_{1} B_{2}-A_{1} A_{2}$. For a trial solution, $C_{2}(t)=e^{i \mu t}$, equation 35 reads,

$$
\mu^{2}+a \mu+b=0
$$

and the general solution can be written as

$$
C_{2}(t)=k_{+} e^{i \mu_{+} t}+k_{-} e^{i \mu_{-} t} \quad\left(\mu_{ \pm}=\left(-a \pm \sqrt{a^{2}-4 b}\right) / 2\right)
$$

If initially at $t=0$, all the atoms were in the lower non-excited states, that is $C_{2}(0)=0$ and $C_{1}(0)=1$, then $k_{+}=-k_{-}=k($ say $)$ and second equation of equation 34 gives

$$
\dot{C}_{2}(0)=i A_{2}=i k\left(\mu_{+}-\mu_{-}\right) \Rightarrow k=\frac{A_{2}}{\left(\mu_{+}-\mu_{-}\right)}=\frac{A_{2}}{\sqrt{a^{2}-4 b}}
$$

Therefore the general solution:

$$
\begin{aligned}
& C_{2}(t)=2 i A_{2} e^{-i a t / 2} \frac{\sin \left(\left(a^{2}-4 b\right)^{1 / 2} t / 2\right)}{\left(a^{2}-4 b\right)^{1 / 2}} \\
& C_{2}(t)=i g U(\boldsymbol{r}) \sqrt{n+1} e^{-i\left(\omega(2 n+3)-2 \omega_{0}\right) t / 2} \frac{\sin \left(\left(\left(\omega-\omega_{0}\right)^{2}+|g|^{2}|U(\boldsymbol{r})|^{2}(n+1)\right)^{1 / 2} \frac{t}{2}\right)}{\left(\left(\omega-\omega_{0}\right)^{2}+|g|^{2}|U(\boldsymbol{r})|^{2}(n+1)\right)^{1 / 2}}
\end{aligned}
$$

Where $a^{2}-4 b=4\left[\left(\omega-\omega_{0}\right)^{2}+|g|^{2}|U(\boldsymbol{r})|^{2}(n+1)\right]$. Since, $|U(\boldsymbol{r})|^{2}=1$, the probability of absorption is

$$
\left|C_{2, n}(t)\right|^{2}=\frac{|g|^{2}(n+1)}{\left(\omega-\omega_{0}\right)^{2}+|g|^{2}(n+1)} \sin ^{2}\left(\sqrt{\left(\omega-\omega_{0}\right)^{2}+|g|^{2}(n+1)} \frac{t}{2}\right)
$$

Similarly by assuming that initially the atom is in the upper energy state and the number of photons present in the cavity is $n$ where the atom transit to lower energy state by emitting a photon, the two quantum states for atom and field can be written as

$$
\left|\psi_{1}\right\rangle=\left|\phi_{2}\right\rangle|n+1\rangle e^{-i\left(E_{1} / \hbar+\omega_{0}\right) t} ; \quad\left|\psi_{2}\right\rangle=\left|\phi_{1}\right\rangle|n\rangle e^{-i E_{1} t / \hbar}
$$

these two equations are almost similar to equations in 24 for absorption, except $\psi_{1}$ and $\psi_{2}$ are interchanged. The initial conditions change to $C_{2}(0)=1$ and $C_{1}(0)=0$. Therefore the probability of emission $\left|C_{1, n+1}(t)\right|^{2}$ is equal to probability of absorption given in equation 40 . Figure 8 shows the variation of the average population of higher energy state $\left|C_{2, n}(t)\right|^{2}$ with the number of photons present in the cavity $|g|^{2} n$. The population increases rapidly at the beginning, but the rate at which 
it increase, reduces dramatically with the increase in the number of photons. According to the equation 40, even when no single photon is present in the cavity, $\left|C_{2, n}(t)\right|^{2}$ is not equal to zero.

Figure 9 represents the behaviour of electron population of excited state with photon number in the laser cavity and time. The maximum population increases as the number of photons increase. The population varies sinusoidal in time, while frequency of sinusoidal pattern increases with the number of photons. Figure 10 represents the variation of population with respect to frequency difference

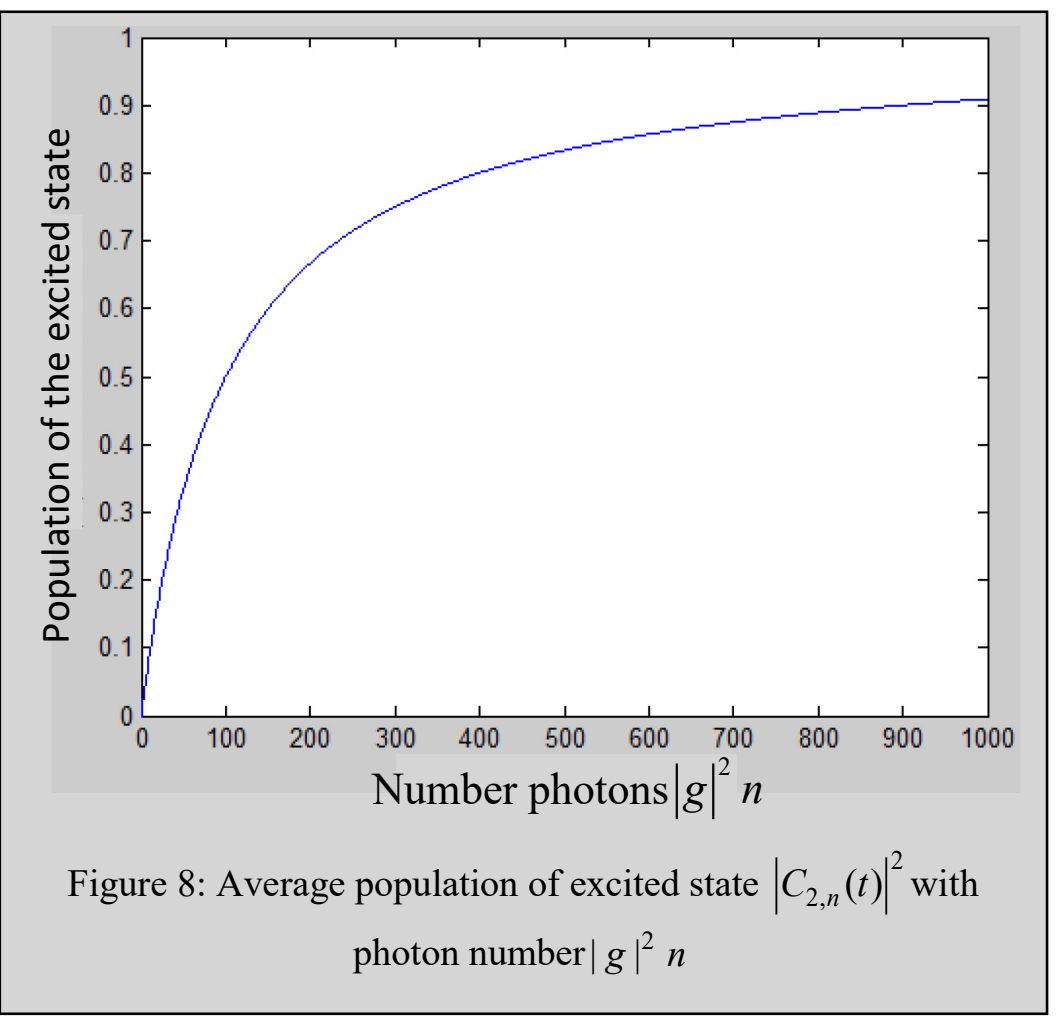
between quantized electric field $\omega$ and resonance frequency $\omega_{0}$ and time. When the frequency of electric field is equal to the resonance frequency of the atom, the population inversion is a maximum. This is similar to the results obtained for the semi-classical theory treating the electric field in the optical cavity classically and the energy states of atoms as quantized [6].

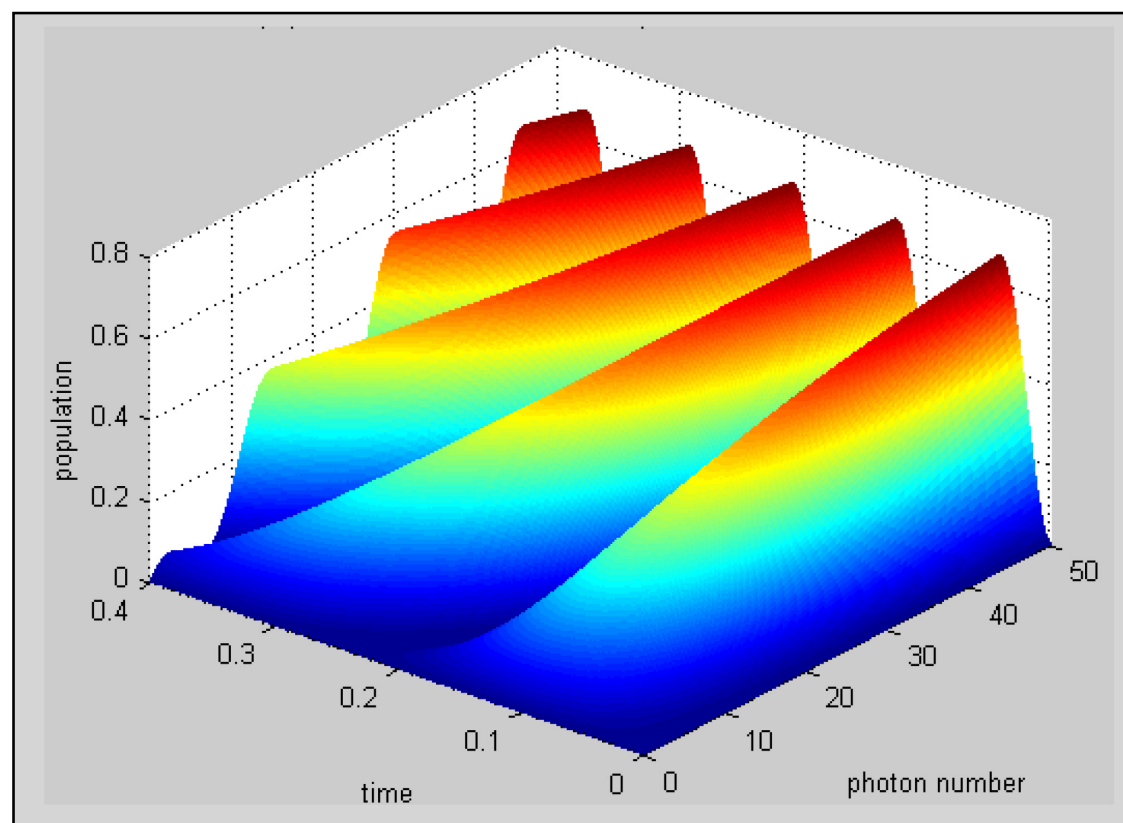

Figure 9: Population of excited state with photon number and time 


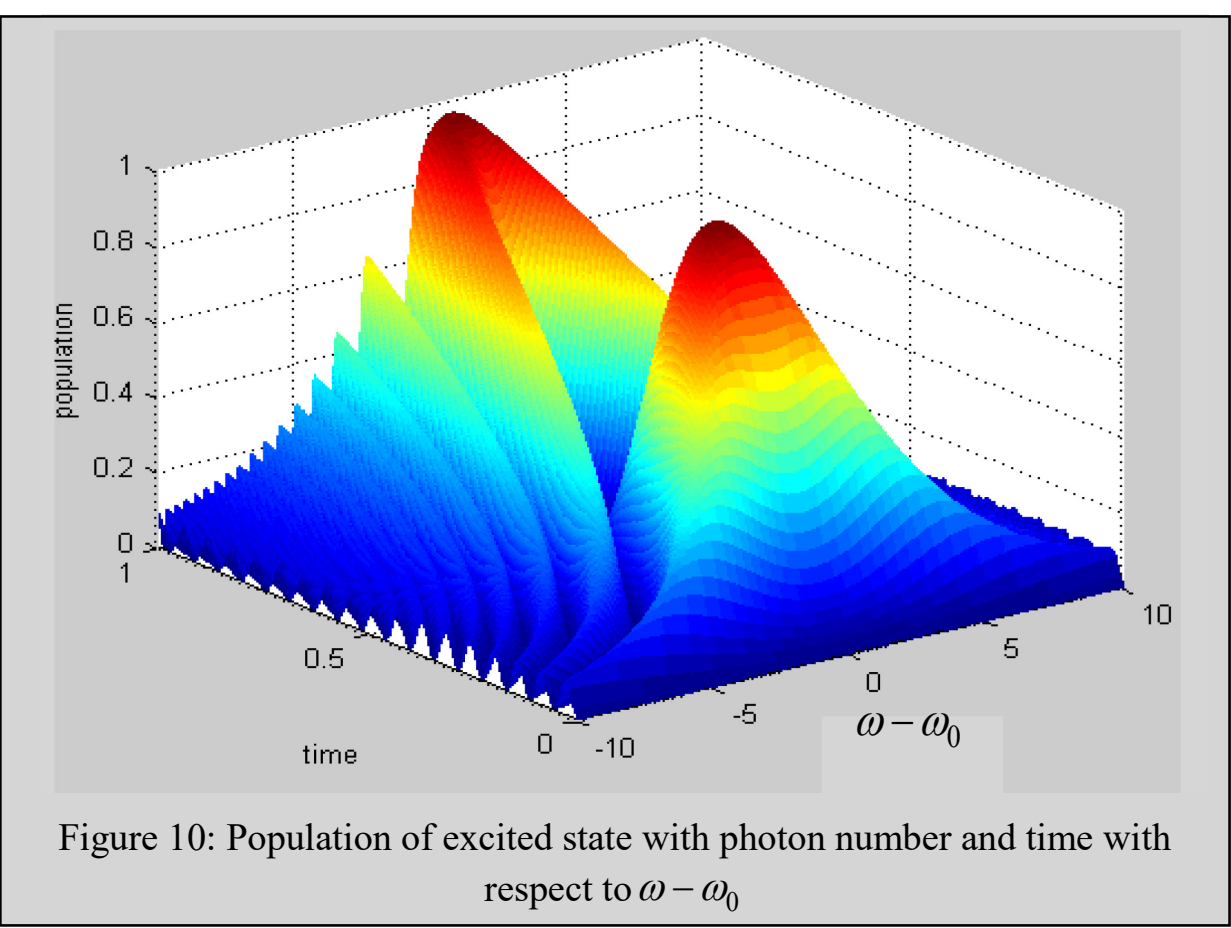

\section{COHERENT STATES}

Mathematically, the coherent stat $|\alpha\rangle$ is defined as the eigenstate of the annihilation operator,

$$
\hat{a}|\alpha\rangle=\alpha|\alpha\rangle
$$

Since $\hat{a}$ is not Hermitian, $\alpha$ is in general a complex number. It is possible to represent the density operator $\hat{\rho}_{F}$ of a single mode laser in the diagonal form

$$
\hat{\rho}_{F}=\int \phi(v, t)|v\rangle\langle v| d^{2} v
$$

where the integral is over the entire complex $v$-plane. Here $|v\rangle$ is the coherent state with complex amplitude $v$ and $\phi(v, t)$ is a real weight function or phase space density, sometimes can be probability density. By replacing creation and annihilation operators by differential operators,

$$
\begin{aligned}
& \hat{a}^{\dagger}|v\rangle\left\langle v\left|=\left(v^{*}+\frac{\partial}{\partial v}\right)\right| v\right\rangle\langle v| \\
& |v\rangle\left\langle v\left|\hat{a}=\left(v+\frac{\partial}{\partial v^{*}}\right)\right| v\right\rangle\langle v|
\end{aligned}
$$

and substituting in to the master equation of the density operator given in equation 14, after some algebra it is possible to obtain [4]

$$
\frac{\partial \phi(\boldsymbol{x}, t)}{\partial t}=-\sum_{i=1}^{2} \frac{1}{2} \frac{\partial}{\partial x_{i}}\left(A-C-B \boldsymbol{x}^{2}\right) x_{i} \phi(\boldsymbol{x}, t)+\frac{1}{4} A \sum_{i=1}^{2} \frac{\partial^{2} \phi(\boldsymbol{x}, t)}{\partial x_{i}^{2}}
$$


which is the Fokker-Plank equation for the phase space density $\phi(\boldsymbol{x}, t)$ for the laser field. Here $\boldsymbol{x}$ is a real two dimensional vector, such that $v=x_{1}+i x_{2}$. Then $x_{1}=\left(v+v^{*}\right) / 2$ and $x_{2}=\left(v-v^{*}\right) / 2$.

Re-scaling the above equation by putting $t \sqrt{A B / 8}=t^{\prime}$ and $(2 B / A)^{1 / 4} x=x^{\prime}$ and dropping the introduced prime signs:

$$
\frac{\partial \phi(\boldsymbol{x}, t)}{\partial t}=-\sum_{i=1}^{2} \frac{\partial}{\partial x_{i}}\left[\left(b-x^{2}\right) x_{i} \phi(\boldsymbol{x}, t)-\frac{\partial \phi(\boldsymbol{x}, t)}{\partial x_{i}}\right]
$$

where $b=(A-C) \sqrt{2 / A B}$. For steady state, $\partial \phi(\boldsymbol{x}, t) / \partial t=0$, since $\phi(\boldsymbol{x}, t)$ is independent of time, the phase space density of steady state $P(x)$ can be taken as $\phi(x, t)$,

$$
\begin{gathered}
\left(b-x^{2}\right) x_{i} P(x)-\frac{\partial P(x)}{\partial x_{i}}=\text { constant }=0 \text { (say) } \Rightarrow P(x)=c e^{-\left(x^{4} / 4-b x^{2} / 2\right)} \\
P(v)=c e^{b^{2} / 4} e^{-\left(|v|^{2}-b\right)^{2} / 4}=a e^{-\left(|v|^{2}-b\right)^{2} / 4} \quad(x=|v|)
\end{gathered}
$$

when $b$ is large, then $P(v)$ has Gaussian distribution in $|v|^{2}$. since $v$ is complex, the phase of $v$ is completely random and uniformly distributed from 0 to $2 \pi$. For a large value of $b$, the mean of $|v|$ and its relative variance are approximately given by

$$
\langle|v|\rangle=\sqrt{b}\left(1-\frac{1}{4 b^{2}}\right) ; \quad \frac{\left\langle(\Delta|v|)^{2}\right\rangle}{\langle|v|\rangle^{2}} \approx \frac{1}{2 b^{2}}
$$

The time dependent Fokker-Plank equation of the phase space density $\phi(x, t)$ given in equation 46 in terms of polar coordinates is,

$$
\frac{\partial \phi(r, \theta, t)}{\partial t}=-r \frac{\partial\left(b-r^{2}\right) \phi(r, \theta, t)}{\partial r}-2\left(b-r^{2}\right) \phi(r, \theta, t)+\left[\frac{\partial^{2}}{\partial r^{2}}+\frac{1}{r} \frac{\partial}{\partial r}+\frac{1}{r^{2}} \frac{\partial^{2}}{\partial \theta^{2}}\right] \phi(r, \theta, t)
$$

By method of separation of variables, the solution of the above equation can be written as,

$$
\phi(r, \theta, t)=\sum_{n=0}^{\infty} \sum_{\beta=-\infty}^{\infty} K_{n \beta} e^{-\lambda_{n \beta} t} e^{-i \beta \theta} \frac{1}{\sqrt{r}} e^{-\left(r^{2}-b\right)^{2} / 8} \psi_{n \beta}(r)
$$

where $\psi_{n \beta}(r)$ are the solutions satisfying equation:

$$
\begin{aligned}
& -\frac{\partial^{2} \psi_{n \beta}(r)}{\partial r^{2}}+V_{\beta}(r) \psi_{n \beta}(r)=\lambda_{n \beta} \Psi_{n \beta} \psi_{n \beta}(r) \\
& V_{\beta}(r)=-\frac{3}{4} r^{6}+6 b r^{4}-\left(4+b^{2}\right) r^{2}+\left(\beta^{2}-\frac{3}{4}\right) \frac{1}{r^{2}}+4 b
\end{aligned}
$$




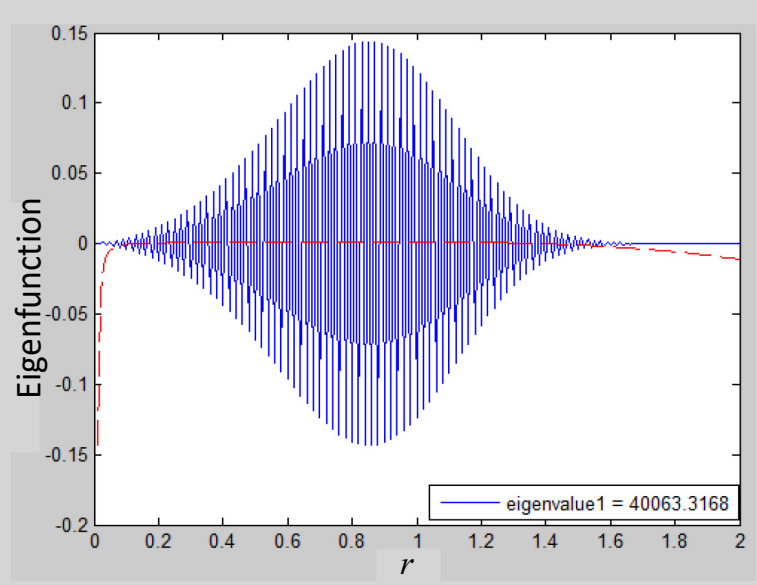

$11(a)$

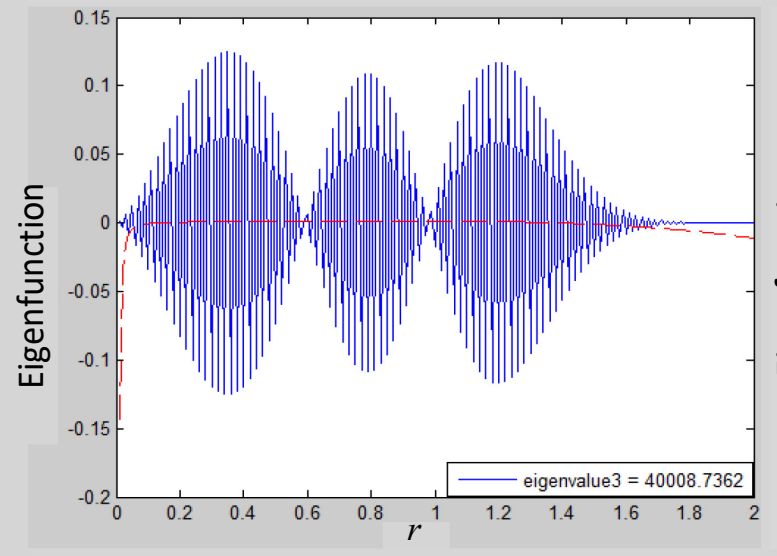

$11(c)$

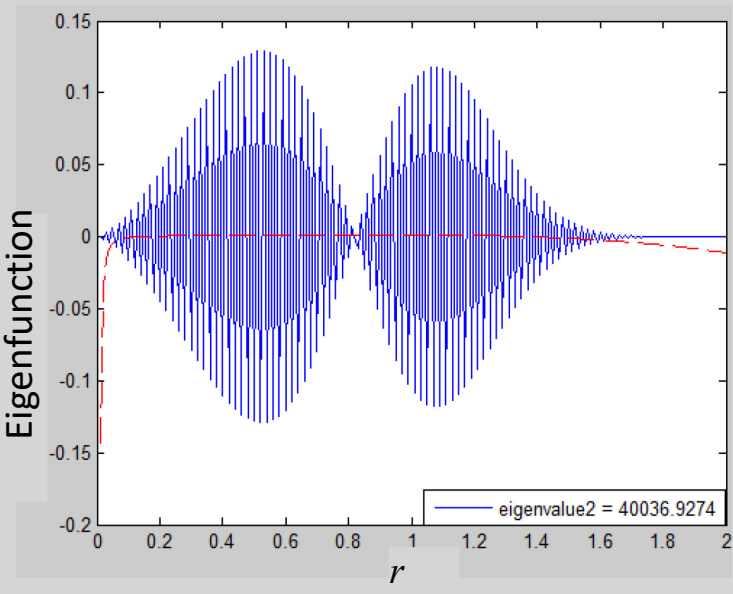

$11(b)$

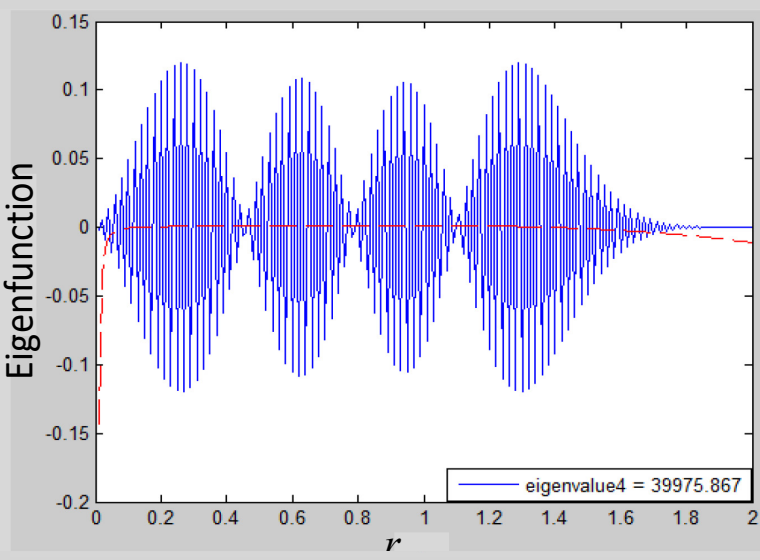

$11(d)$

Figure 11: Eigenfunctions corresponding to four largest eigenvalues $(a)$ 40063, (b) 40037, (c) 4009 and $(d) 39976$ for $\beta=0$ and $b=10$ represented in blue. The red line represents the variation of the potential function $\boldsymbol{V}(\boldsymbol{r})$

The figure 11 represents the eigenfunctions corresponding to the largest positive eigenvalue 40063 ,

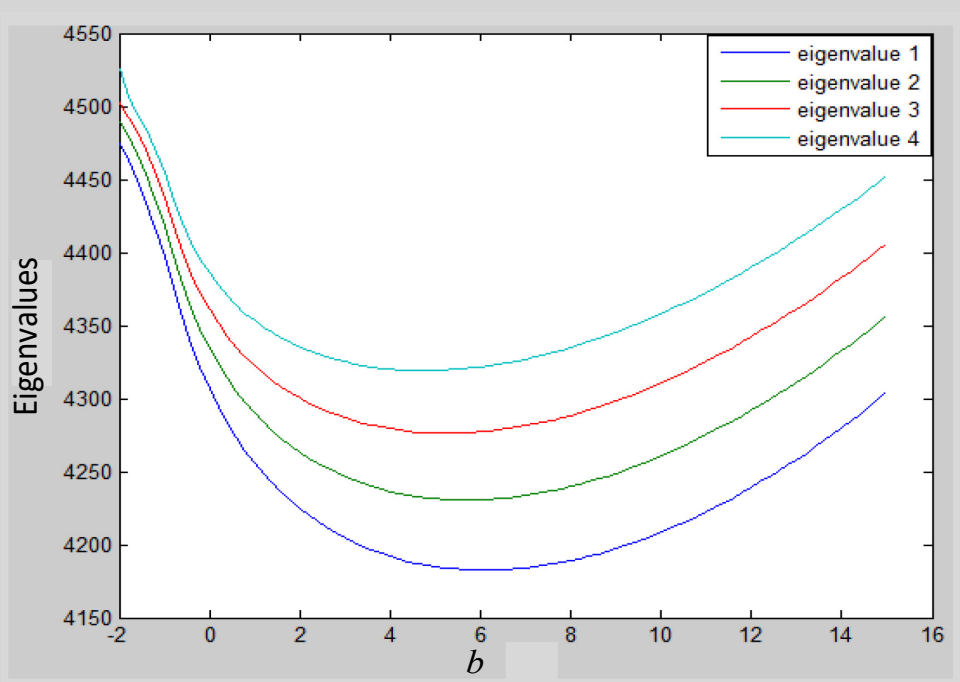

Figure 12: The first four eigenvalues with the pump parameter $b$ 40037, 4009 and 39976 of equation 52 when $b=10$ and $\beta=0$. The red line represents the potential function $V(r)$. Figure 12 represents the first four eigenvalues for pump parameter in the range $b=-2$ to 16 . The plot was obtained for the range $r=0.1$ to 2.0 . The paths of eigenvalues separate from each other, and all have a minimum in the positive rage of the pump parameter around six. Figure 13 
represents the variation of eigenvalues for the pump parameters $-5,0,5,10$. The plot was obtain for the range $r=0.1$ to $r=2$, and for 100 eigenvalues. For different pump parameters in the range $b=-5$ to 15 hundred eigenvalues are presented in the three dimensional figure 14 .
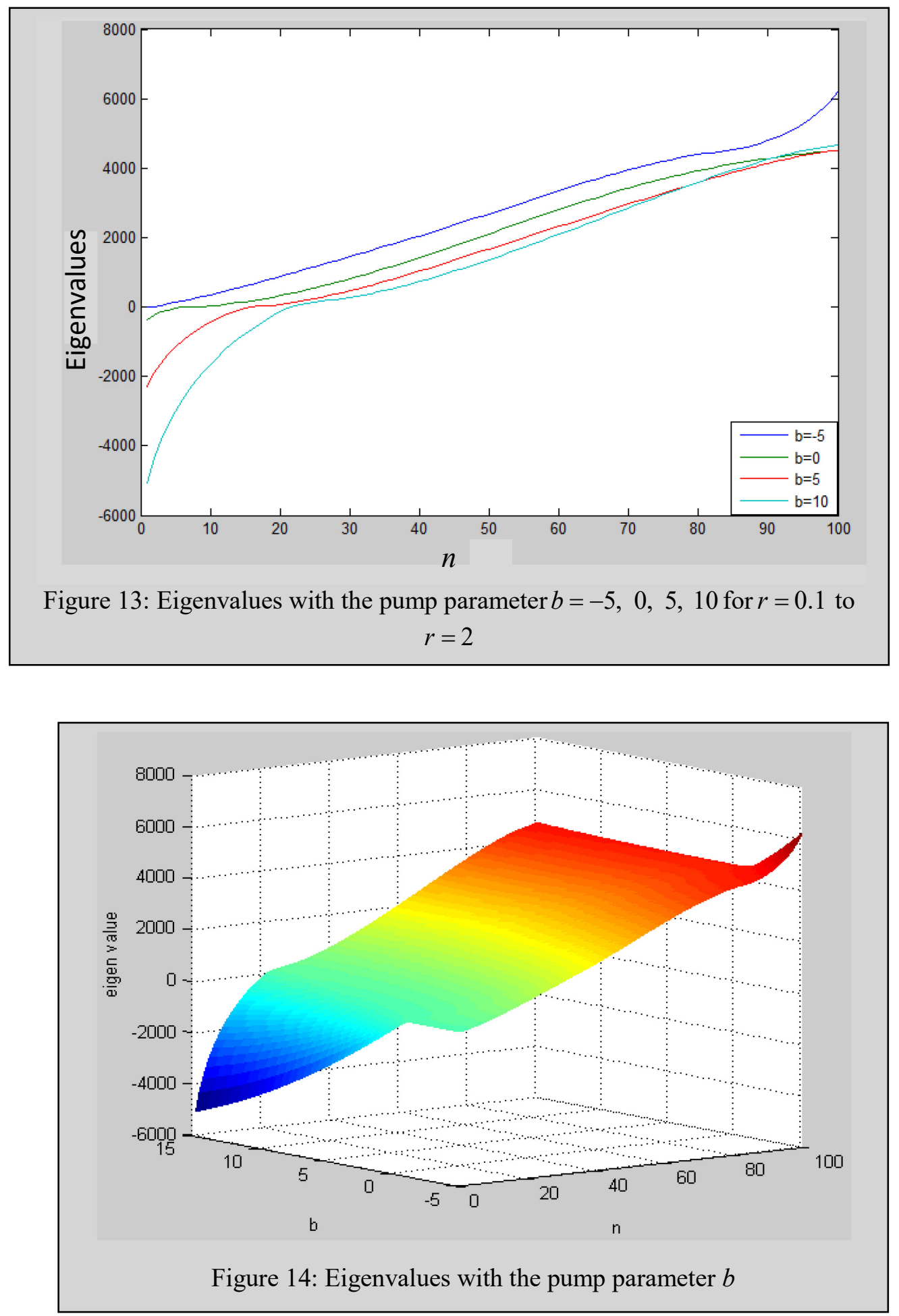

\section{CONCLUSIONS}

Quantum mechanical and coherence state models were used to study the field inside a laser cavity. There is the probability of emission even when no single photon is present in the system for the quantum model. This can be attributed to stimulated emission and to the spontaneous emission. 
The spontaneous emission does cause some light to be emitted under all conditions and thus does not create a discontinuity at the origin. The quantum theory model gives a clear understanding of absolute strength of the laser field and also provides a description of spontaneous emission in lasers. In the coherent state model, the phase space density or probability density of coherent state has a Gaussian distribution. But for large values of pump parameter, the phase density becomes narrower as the parameter increases while phase remains random. A field above a threshold can be represents by a randomly phased mixture of coherent states. The increase of pump parameter makes the phase density of laser field narrower and tends toward a delta function, which suggests that the laser field above threshold can be considered as in coherent state. The coherent state treatment gives a clear description of laser characteristics and how these alter with various conditions.

\section{References}

[1] A. Einstein, Physikalische Gesellschaft Zürich 18 (1917)121-128.

[2] J. P. Gordon, H. J. Zeiger, and C. H. Townes, Phys. Rev. 95 (1954) 282

[3] R. J. Glauber Phys. Rev. 131(6) (1963) 2766-2788.

[4] L.Mandel, E.Wolf, Optical Coherence and Quantum Optics (Cambridge University Press. 1995) pp.522-570, 741-760, 900-950.

[5] M. Banaee, Coherent states in laser physics (University of British Colombia, 2004)

[6] K.M Priyashanka, K.A.I.L.Wijewardena Gamalath WSN 7 (2015) 101-115. 